\title{
Os Efeitos da Idade na Percepção Visual de Grades Senoidais em Luminância Baixa
}

\section{The Effects of Age on Visual Perception of Sine-Wave Gratings at Low Luminance}

\author{
Natanael Antonio dos Santos ${ }^{\mathrm{a} *}$, Adriana Bastos Oliveira ${ }^{\mathrm{a}}$, Renata Maria Toscano Barreto Lyra \\ Nogueira $^{a}$, Éllen Dias Nicácio da Cruz ${ }^{\mathrm{a}}$, Maria Lúcia de Bustamante Simas ${ }^{\mathrm{b}}$ \\ ${ }^{a}$ Universidade Federal da Paraíba, João Pessoa, Brasil, ${ }^{b}$ Universidade Federal de Pernambuco, Recife, Brasil
}

\begin{abstract}
Resumo
O objetivo deste trabalho foi investigar a função de sensibilidade ao contraste (FSC) de adultos e idosos (20-23, 30-39, 40-49, 50-59, 60-69 anos) para grades senoidais de 0,25 a 2 cpg em luminância baixa. Todos os participantes apresentavam acuidade visual normal e se encontravam livres de doenças oculares identificáveis. Foram estimados limiares de contraste para 30 participantes (seis em cada faixa etária) utilizando o método psicofísico da escolha forçada. Neste método, os participantes tinham que escolher um estímulo contendo uma freqüência de teste (grade senoidal) em baixo contraste ou um estímulo neutro com luminância média de $0,7 \mathrm{~cd} /$ $\mathrm{m}^{2}$. Os resultados mostraram que os grupos de 50-59 e 60-69 anos apresentaram prejuízos significativos na FSC nas freqüências espaciais de 1 e $2 \mathrm{cpg}$ comparados ao grupo de 20-23 anos. Estes resultados sugerem alterações relacionadas à idade na FSC de freqüências espaciais em níveis baixos de luminância.

Palavras-chave: Percepção visual; sensibilidade ao contraste; envelhecimento; freqüência espacial; método da escolha forçada.
\end{abstract}

\begin{abstract}
The aim of this work was to investigate the contrast sensitivity function for sine-wave gratings in the range between 0.25-2 cpd in young and older adults (20-23, 30-39, 40-49, 50-59, 60-69 years old) using low luminance. All subjects were free from identifiable ocular disease and had normal acuity. We measured the contrast thresholds for 30 participants (six volunteers in each age) using the psychophysical forced-choice staircase method. In this method the volunteers had to choose the stimulus containing a test frequency at low contrast (e.g., a sine-wave grating), or another neutral stimulus at mean luminance $\left(0.7 \mathrm{~cd} / \mathrm{m}^{2}\right)$. The results showed significant changes of CSF for sinewave gratings at 1 and $2 \mathrm{cpd}$ for the adults 50-59 and 60-69 years old compared to the adults of 2023 years old. These results suggest age-related changes in the CSF for sine-wave grating at low luminance levels.

Keywords: Visual perception; contrast sensitivity; aging; spatial frequency; forced-choice staircase method.
\end{abstract}

Pesquisas psicofísicas e neurofisiológicas defendem a idéia proposta originalmente por Campbell e Robson (1968) de que o sistema visual humano (SVH) é composto por mecanismos ou canais que respondem seletivamente e simultaneamente a bandas estreitas de frequiências espaciais (Santos \& Simas, 2002; Santos, Simas \& Nogueira, 2003, 2004; Simas \& Santos, 2002a, 2002b; Wilkinson et al., 2000; Wilson, Levi, Maffei, Rovamo \& De Valois, 1990). Ou seja, a abordagem de canais múltiplos pressupõe que a forma dos objetos é decomposta ou analisada, inicialmente, pela retina em componentes elementares ou freqüências espaciais. E em seguida, estas informações são conduzidas através de vias visuais que passam pelo núcleo geniculado lateral e

\footnotetext{
Endereço para correspondência: Departamento de Psicologia, CCHLA - UFPB. Campus I, Cidade Universitária, Conjunto Castelo Branco, João Pessoa - PB, 58051-900. Fone: (83) 3216 7006; Fax: (83) 3216-7064. Email: natanael_labv@yahoo.com.br / natanael.santos@pesquisador.cnpq.br Suporte financeiro: CNPq e FACEPE.
}

se projetam no córtex visual, onde ocorre o processo de recomposição ou síntese e, naturalmente, a detecção e/ou reconhecimento do objeto (R. L. De Valois \& K. K. De Valois, 1988; O’Donnell et al., 2002). Esses estudos partem do conhecimento de que a função de sensibilidade ao contraste (FSC) representa o envelope da sensibilidade para a série total de canais (vias), cada um sensível a uma região do espectro (Braddick, Campbell \& Atkinson, 1978; Graham, 1972; Graham \& Nachmias, 1971). Em outras palavras, a mensuração da FSC permite estimar o limiar ou a quantidade mínima de contraste que o SVH precisa para detectar individualmente cada faixa de freqüência espacial. Freqüência espacial é o número de ciclos (listras claras e escuras) por grau de ângulo visual (cpg). Enquanto, contraste é a relação entre a diferença da luminância máxima (listra clara) e luminância mínima (listra escura) dividido pela soma das duas.

Entre as correntes de modelos de canais múltiplos, Wilson et al. propuseram um modelo com seis canais capazes de 
varrer uma faixa de frequêencia 0,25 a $22 \mathrm{cpg}$ (Wilson \& Gelb, 1984; Wilson, McFarlane \& Philips, 1983). Segundo estes autores, os seis canais teriam picos de sensibilidade por volta de 0,$8 ; 1,5 ; 2,8 ; 4,4 ; 8,0$ e $16 \mathrm{cpg}$, respectivamente. Outras informações sobre modelos de canais múltiplos podem ser encontradas em Wilson et al. (1990).

Uma característica importante da FSC advém do fato que a sua forma muda em função das condições de visualização (Benedek, Benedek, Kéri \& Janáky, 2003; R. L. De Valois \& K. K. De Valois, 1988; Georgeson \& Sullivan, 1975), do período de desenvolvimento da visão (Candy, Crowell \& Banks, 1998; Courage \& Adams, 1996; Van Sluyters, Atkinson, Held, Hoffman \& Shatz, 1990) e de alterações neuropatológicas (Akutsu \& Legge, 1995; Bour \& Apkarian, 1996; Elliott \& Situ, 1998; O'Donnell et al., 2002; Slaghuis \& Thompson, 2003; Suttle \& Tumer, 2004; Ventura et al., 2005), o que permite fortes inferências sobre o funcionamento de mecanismos fisiológicos básicos relacionados ao processamento visual de objeto. Por essas e outras características, a FSC é considerada uma das técnicas mais completas e poderosas na avaliação teórica e clínica das funções visuais (Adams \& Courage, 2002; John, 1997; Kiper \& Kiorpes, 1994; Montés-Micó \& Ferrer-Blasco, 2001; Richman \& Lyons, 1994; Van Sluyters et al., 1990; Wilson et al., 1990).

Neste contexto, a FSC tem sido utilizada, ao longo das últimas décadas, para investigar a existência de alterações significativas nas características neurais e ópticas do SVH relacionadas ao envelhecimento (Arden, 1978; Arden \& Jacobsen, 1978; McGrath \& Morrison, 1981; Owsley, Sekuler \& Siemsen, 1983; Sekuler, Hutman \& Owsley, 1980; Skalka, 1980). Entretanto, os estudos realizados não originaram resultados convergentes. Por exemplo, Arden (1978) mensurou a FSC para freqüências espaciais baixas e médias em participantes de 11 a 70 anos, utilizando placas fotográficas. Cada placa de Arden continha uma freqüência espacial específica e contraste que variava de um nível sublimiar, na parte superior, a um nível supralimiar, na parte inferior da placa, o que permitia ao pesquisador descobrir lentamente a placa até que o participante detectasse a grade. Os resultados de Arden não revelaram mudanças na FSC relacionadas ao processo de envelhecimento em nenhuma frequiência espacial testada.

Por sua vez, Arden e Jacobsen (1978), utilizando o mesmo aparato, procedimento e amostra semelhante, encontraram sensibilidade levemente reduzida para os voluntários mais velhos em todas as frequiências espaciais testadas. Resultados semelhantes foram encontrados por outros autores com as placas de Arden (Skalka, 1980; Sokol, Domar \& Moskowitz, 1980) e com osciloscópio e o método psicofísico do ajustamento (McGrath \& Morrison, 1981).

Por outro lado, Sekuler et al. (1980) mensuraram a FSC para adultos e idosos com idade média de 18 e 73 anos. O limiar para cada grade senoidal foi mensurado com o método psicofísico do ajustamento. As frequêencias espaciais de 0,$5 ; 1,0 ; 2,0 ; 4,0 ; 8,0$ e $16 \mathrm{cpg}$ foram apresentadas em um monitor com luminância média de $55 \mathrm{~cd} / \mathrm{m}^{2}$ controlado por um microcomputador. Os resultados encontrados mostraram sensibilidade menor para freqüências espaciais baixas e médias no grupo de idosos comparado ao grupo de jovens. Os autores não encontraram diferenças entre o grupo de jovens e idosos para freqüências altas (p.ex., $16 \mathrm{cpg}$ ).

Outros, contrários aos estudos citados acima, relatam que o envelhecimento afeta principalmente a sensibilidade a freqüências espaciais mais altas (Arundale, 1978; Derefeldt, Lennerstrand \& Lundh, 1979). Por exemplo, Arundale mensurou a FSC de adultos jovens e idosos para freqüências espaciais de 0,25 a $28 \mathrm{cpg}$ com o método psicofísico dos limites. Os limiares foram estimados com grades senoidais apresentadas em um televisor com luminância média de $100 \mathrm{~cd} / \mathrm{m}^{2}$. Os resultados demonstraram que os voluntários com idade acima de 60 anos apresentaram redução na FSC nas freqüências espaciais acima de $2,0 \mathrm{cpg}$ quando comparados aos grupos mais jovens.

Finalmente, alguns trabalhos relatam que o envelhecimento afeta principalmente as freqüências espaciais médias e altas (Crassini, Brown \& Bowman, 1988; Elliott, 1987; Higgins, Jaffe, Caruso \& Monasterio, 1988; Owsley et al., 1983; Ross, Clarke \& Bron, 1985). Por exemplo, Owsley et al. mensuraram a FSC de adultos e idosos em sete faixas etárias (19-28, 31-38, 41-48, 50-58, 60-69, 70-79 e 80-87 anos), utilizando luminância média de $103 \mathrm{~cd} / \mathrm{m}^{2}$ e freqüências espaciais de 0,$5 ; 1 ; 2 ; 4 ; 8$ e $16 \mathrm{cpg}$. Os resultados mostraram que a sensibilidade ao contraste para frequiências espaciais baixas (p. ex., 0,5 e $1 \mathrm{cpg}$ ) permanece a mesma em todas as idades. Por outro lado, a sensibilidade ao contraste para freqüências espaciais médias e altas $(2-16 \mathrm{cpg})$ diminui com a idade a partir da faixa etária 41-48 anos. Este declínio tornou-se mais acentuado com o aumento da idade, de forma que o grupo de voluntários acima de 60 anos apresentou perdas estatisticamente significantes na sensibilidade ao contraste para as freqüências espaciais médias e altas comparado ao grupo mais jovem.

Em termos gerais, não existe um consenso sobre as faixas de freqüências que são afetadas pelo envelhecimento. Embora, os estudos mais recentes relatam que o processo de envelhecimento reduz a sensibilidade ao contraste principalmente nas freqüências espaciais médias e altas (Fiorentini, Porciatti, Morrone \& Burr, 1996; Santos et al., 2004; Schefrin, Tregear, Harvey Jr. \& Werner, 1999).

\section{O Presente Estudo}

Este estudo dá continuidade aos experimentos que procuraram caracterizar a FSC de adultos e idosos em faixas etárias distintas. Entretanto, diferente da maioria dos estudos encontrados na literatura que utilizam níveis altos de luminância, nós utilizamos luminância baixa. As principais premissas para estudos desta natureza vêm (a) da necessidade de especificar alterações no processamento visual de contraste (ou frequiência espacial) relacionadas à idade; (b) da necessidade de acompanhar e descrever o funcionamento de mecanismos neurofisiológicos básicos da percepção visual e (c) da necessidade de testar e desenvolver técnica psicofísica capaz de caracterizar e acompanhar a 
resposta do SVH com e sem patologias. Neste sentido, partindo do pressuposto que a FSC muda gradativamente com o envelhecimento e que a maioria dos estudos encontrados na literatura utiliza níveis altos de luminância, o presente estudo teve como objetivo mensurar a FSC de adultos e idosos em cinco faixas etárias (20-23, 30-39, 40-49, 50-59 e 60-69 anos), utilizando o método psicofísico da escolha forçada e estímulos visuais do tipo grade senoidal vertical acromática em níveis baixos de luminância $\left(0,7 \mathrm{~cd} / \mathrm{m}^{2}\right)$.

A luminância de $0,7 \mathrm{~cd} / \mathrm{m}^{2}$, geralmente, é considerada na literatura como mesópica (Charman, 1996; Montés-Micó, Espana, Bueno, Charman \& Menezo, 2004), embora exista trabalho que a descreva como fotópica baixa (Van de Grind, Koenderink \& Van Doorn, 2000). Neste nível de luminância é possível avaliar a resposta mutua das vias visuais parvocelular e magnocelular. A via parvocelular é especializada no processamento de freqüências espaciais médias e altas (detalhes finos dos objetos) e opera em níveis altos, fotópicos de luminância. Já a via magnocelular é especializada no processamento de frequiências espaciais baixas e opera em níveis baixos, escotópicos de luminância (Benedek et al., 2003; Ellemberg, Lewis, Liu \& Maurer, 1999).

\section{Método}

\section{Participantes}

Participaram do estudo 30 voluntários de ambos os sexos, seis participantes em cada uma das cinco faixas etárias [20-23 anos $(\mathrm{M}=21,7 ; \mathrm{DP}=0,84), 30-39$ anos $(\mathrm{M}=35,2$; $\mathrm{DP}=2,59), 40-49$ anos $(\mathrm{M}=44,6 ; \mathrm{DP}=2,88), 50-59$ anos $(\mathrm{M}=54,86 ; \mathrm{DP}=2,47)$ e $60-69$ anos $(\mathrm{M}=63,60 ; \mathrm{DP}=1,67)]$. Todos os participantes apresentavam acuidade visual normal ou corrigida e tinham consultado os seus oftalmologistas há menos de 12 meses. Os mesmos também estavam livres de doenças oculares identificáveis. A participação na pesquisa ocorreu mediante a assinatura de termo de consentimento livre e esclarecido e conforme a Resolução ${ }^{\circ}$ 196/96 do Conselho Nacional de Saúde, que trata das diretrizes e normas de pesquisas envolvendo seres humanos. Projeto cadastrado na Comissão Nacional de Ética em Pesquisa do Ministério da Saúde (CONEP/MS-064608) e aprovado pelo Comitê de Ética local.

\section{Equipamentos e Estímulos}

Os estímulos foram gerados em um monitor de vídeo CLINTON MEDICAL monocromático de 21 polegadas, digital, de alta resolução, controlado por um microcomputador. Uma cadeira foi fixada a $150 \mathrm{~cm}$ da tela do monitor de vídeo. A luminância média da tela foi de 0,7 $\mathrm{cd} / \mathrm{m}^{2}$ ajustada por um fotometro do tipo SPOT METTER ASAHI PENTAX com precisão de um grau. A luminância mínima da tela foi de $0,5 \mathrm{~cd} / \mathrm{m}^{2}$ e a máxima de $0,9 \mathrm{~cd} / \mathrm{m}^{2}$.

Foram utilizados estímulos do tipo grade senoidal vertical com frequêencias espaciais de 0,$25 ; 0,5 ; 1$ e 2 ciclos por grau de ângulo visual, cpg (ver Figura 1). Estes eram circulares com um diâmetro de 7 graus de ângulo visual a 150 $\mathrm{cm}$ de distância da tela. Os mesmos foram gerados em tons de cinza e apresentados em tempo real no monitor.
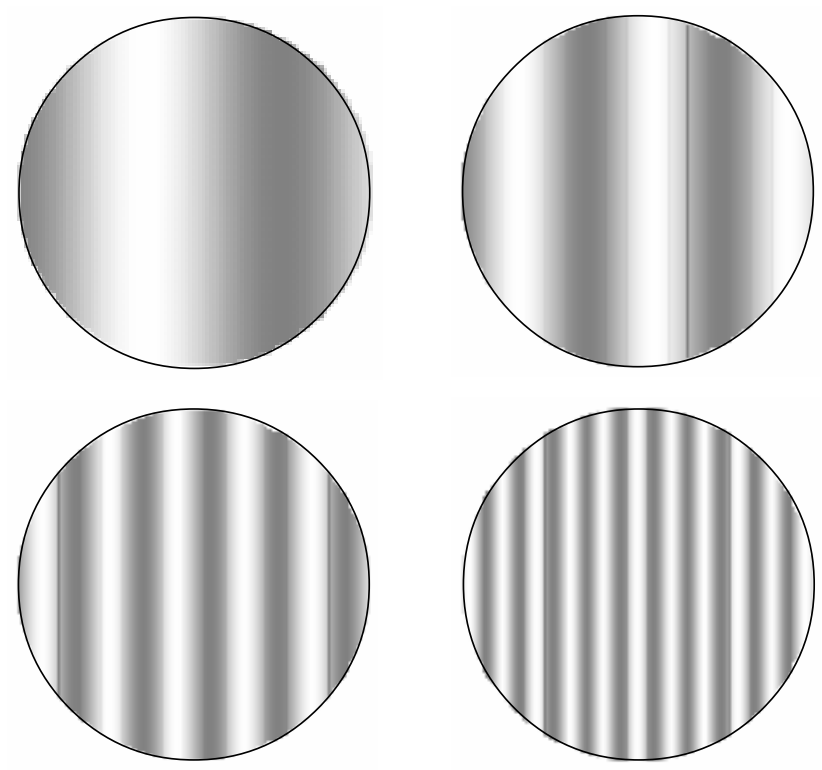

Figura 1. Exemplos de estímulos de freqüências espaciais (acima, da esquerda para a direita, 0,25 e 0,5 cpg e embaixo, da esquerda para direita, 1 e $2 \mathrm{cpg}$ ). Estímulos originalmente calibrados para serem vistos a $150 \mathrm{~cm}$ de distância.

\section{Procedimento}

As estimativas foram realizadas com método psicofísico da escolha forçada (Santos et al., 2003, 2004; Santos, Nogueira \& Simas, 2005; Simas \& Santos, 2002a, 2002b; Wetherill \& Levitt, 1965). Este método se baseia no cálculo da probabilidade de acertos consecutivos por parte dos participantes, ou seja, em cerca de 100 apresentações de escolhas entre os dois estímulos, a frequêencia espacial (estímulo de teste) é percebida $79 \%$ das vezes pelo voluntário.

O procedimento para medir o limiar para cada freqüência consistiu na apresentação sucessiva simples do par de estímulos e o participante teve que escolher dentre eles qual continha a freqüência espacial. O outro estímulo (estímulo neutro) foi sempre um padrão homogêneo com luminância média de $0,7 \mathrm{~cd} / \mathrm{m}^{2}$. O critério adotado para variar o contraste de cada frequência espacial testada era o de três acertos consecutivos para decrescer uma unidade e um erro para acrescer da mesma unidade $(0,08 \%)$.

Durante cada sessão experimental era apresentada uma seqüência de estímulos que foi iniciada com um sinal sonoro seguido imediatamente pela apresentação do primeiro estímulo por $2 \mathrm{~s}$ e de um intervalo entre estímulos de $1 \mathrm{~s}$, seguido pela apresentação do segundo estímulo por $2 \mathrm{~s}$ e da resposta do participante. A ordem de apresentação dos estímulos foi aleatória. Se a resposta do voluntário fosse correta, era seguida por outro sinal sonoro e um intervalo de $3 \mathrm{~s}$ para a sequiência se repetir. $O$ sinal sonoro que indicava o início da apresentação do par de estímulos e o que indicava a escolha correta eram diferentes. A sessão experimental variava em duração dependendo dos erros e acertos do participante até proporcionarem um total de dez reversões (cinco máximos e cinco mínimos) conforme requerido para o final automático da mesma. 
Cada um dos pontos (ou freqüências) da curva de sensibilidade ao contraste foi estimado pelo menos duas vezes (duas sessões experimentais), em dias diferentes, por cada um dos participantes. No total, 72 curvas foram medidas, sendo 12 curvas para cada faixa etária ou grupo de voluntários, gerando uma amostra de aproximadamente 120 valores para cada um dos pontos estimados. Todas as estimativas foram medidas à distância de $150 \mathrm{~cm}$, com visão binocular.

Os participantes pressionavam o botão esquerdo do mouse quando a freqüência espacial era apresentada primeiro e o botão direito quando era apresentada em segundo lugar, isto é, após o estímulo neutro. Os experimentos só começaram quando o experimentador certificou-se que o participante entendeu as instruções.

\section{Resultados}

Os valores mínimos e máximos de contrastes obtidos para cada ponto foram agrupados em planilhas por freqüência espacial e por faixa etária (20-23, 30-39, 40-49, 50-59 e 60-69 anos) e a grande média foi utilizada como estimativa do limiar de contraste. A sensibilidade ao contraste (FSC) é o inverso do limiar de contraste (1/FSC). Assim, quanto menor o limiar de contraste maior a sensibilidade do SVH e vice-versa. Isto é, os menores valores de limiares correspondem aos maiores valores de sensibilidade ao contraste (Figura 2).
A Figura 2 mostra a FSC (média geral) para cada uma das cinco faixas etárias (20-23, 30-39, 40-49, 50-59 e 6069 anos). As barras verticais indicam os erros padrões das médias com intervalos de $99 \%$ de confiança corrigidos para o tamanho da amostra pelo estatístico t-Student. A faixa de sensibilidade máxima ocorreu entre as freqüências espaciais de 0,25-0,5 cpg para todos os grupos.

Os valores de contrastes máximos e mínimos foram submetidos à análise de variância (ANOVA) e a análise post hoc utilizando o teste Tukey HSD (nível de significância $\mathrm{p}=0,05)$. A comparação da idade versus freqüências espaciais através da ANOVA permitiu observar efeito principal significante de idade, $\mathrm{F}_{(4,2399)}=43,84(\mathrm{p}<0,001)$, um efeito principal significativo de frequiência espacial, $\mathrm{F}_{(3,2399)}=$ 133,19 (p<0,001), e um interação significante de idade versus frequiência espacial, $F_{(12,2399)}=104,41(p<0.001)$. A análise post-hoc (teste Tukey HSD) revelou que a FSC do grupo de 20-23 anos é estatisticamente menor do que a FSC do grupo de 50-59 anos nas frequiências espaciais de $1 \mathrm{cpg}$ $(\mathrm{p}<0,001)$ e $2 \mathrm{cpg}(\mathrm{p}<0,001)$ e do que a FSC do grupo de 60-69 anos nas freqüências espaciais de $0,5 \mathrm{cpg}(\mathrm{p}<0,001)$, $1 \mathrm{cpg}(\mathrm{p}<0,001)$ e $2 \mathrm{cpg}(\mathrm{p}<0,001)$. Por outro lado, não encontramos diferenças significantes entre os grupos na freqüência espacial mais baixa (p.ex., $0,25 \mathrm{cpg}$ ).

Os resultados obtidos em níveis baixos de luminância não demonstraram alterações significantes relacionadas à idade na freqüência espacial mais baixa (i.e., $0,25 \mathrm{cpg}$ ). Eles revelaram decréscimos significativos na FSC apenas nas frequiências espaciais mais altas (i.e., 0,$5 ; 1$ e $2 \mathrm{cpg}$ ) e apenas a partir dos 50 anos.

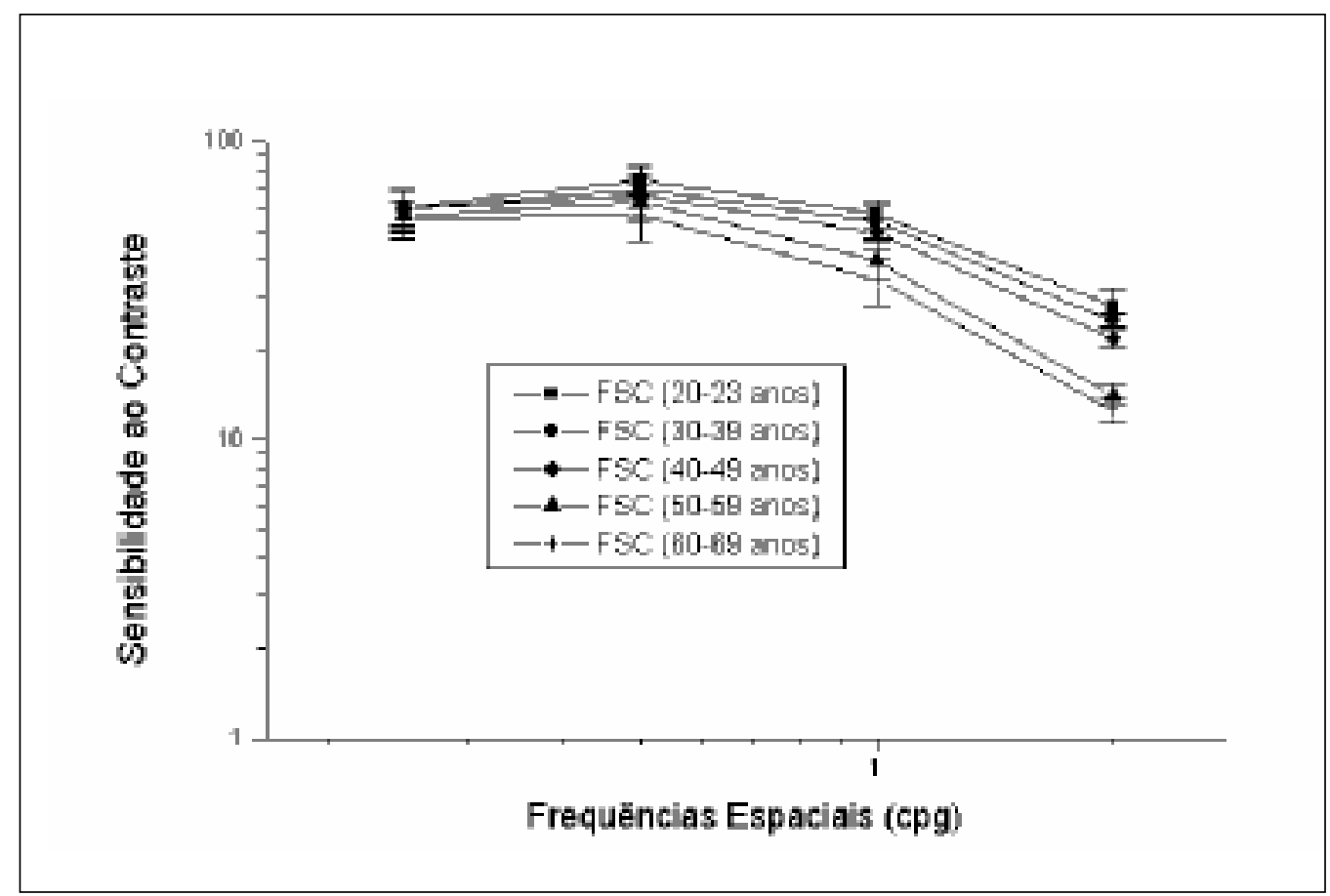

Figura 2. Curvas de sensibilidade ao contraste para freqüências espaciais de adultos e idosos em cinco faixas etárias. As barras verticais indicam os erros padrões das médias com intervalos de $99 \%$ de confiança corrigidos para o tamanho da amostra pelo estatístico t-Student. 


\section{Discussão}

Este trabalho focaliza duas questões básicas relacionadas ao estudo da percepção visual da forma e o envelhecimento. A primeira mensura a FSC de adultos e idosos com o método psicofísico da escolha forçada em níveis baixos de luminância. A segunda investiga o perfil da FSC relacionada à idade, procurando determinar as freqüências (ou faixas de freqüências) mais atingidas pelo envelhecimento.

Os nossos resultados mostram curvas de sensibilidade ao contraste para freqüências espaciais $(0,25 ; 0,5 ; 1$ e 2 cpg) em baixa luminância para 30 participantes divididos em cinco faixas etárias (20-23, 30-39, 40-49, 50-59 e 60-69 anos). As curvas (FSCs) são consistentes uma vez que elas apresentam perfis gerais muito semelhantes. As diferenças entre as curvas (FSCs) podem ser traduzidas no domínio da sensibilidade ao contraste que diminui gradativamente com o aumento da idade (Figura 2). Isto é, o aumento da idade leva o sistema visual a precisar de mais contraste para detectar freqüências espaciais. Entretanto, as alterações produzidas na FSC pelo aumento da idade não se distribuem igualmente pelo espectro de freqüências espaciais. Os declínios observados entre os grupos na FSC não foram significativos na freqüência espacial mais baixa (p.ex., 0,25 cpg) em nenhuma das faixas etárias investigadas (Figura 2). Alterações significantes ocorreram nas freqüências espaciais de 1 e 2 cpg a partir dos 50 anos. Diferenças estas que foram mais acentuadas para os participantes acima dos 60 anos nas frequiências espaciais de 0,5; 1 e 2 cpg. Embora, os nossos resultados tenham sido obtidos com luminância baixa, eles são semelhantes aos dados psicofísicos obtidos com luminâncias altas que não encontraram diferenças na FSC de freqüências espaciais abaixo de $0,5 \mathrm{cpg}$ (Arundale, 1978; Crassini et al., 1988; Derefeldt e et al., 1979; Elliott, Whitaker \& MacVeigh, 1990; Fiorentini et al., 1996; Higgins et al., 1988; Owsley et al., 1983; Ross et al., 1985) e se diferenciam daqueles que relataram diferenças entre adultos e idosos nas freqüências espaciais baixas (McGrath \& Morrison, 1981; Sekuler et al., 1980; Skalka, 1980; Sokol et al., 1980). Este estudo também mostrou declínio na FSC a partir dos 50 anos, conforme discutido por aqueles estudos que utilizaram luminâncias altas (Higgins et al., 1988; Nio et al., 2000; Owsley et al.,1983; Santos et al., 2004).

É possível que parte das divergências encontradas na literatura estejam relacionadas a questões metodológicas e até mesmo ao estado de saúde da amostra. Por exemplo, estudos realizados com as placas de Arden podem ser influenciados pela iluminação e pela velocidade com que cada grade é descoberta pelo experimentador (Owsley et al., 1983). Assim como, já se sabe que doenças comuns da terceira idade (glaucoma, catarata, diabetes, dentre outras) afetam diferentemente a sensibilidade ao contraste (Woods, Tregear \& Mitchell, 1998). Não está claro em alguns experimentos mencionados no início se todos os voluntários apresentavam boa acuidade visual e estavam livres de doenças ou patologias oculares significantes.

Além do mais, parte dos estudos não fornece a luminância da tela ou as condições de visualização dos estímulos (p.ex.,
Skalka, 1980). A luminância é importante porque a FSC depende das condições de visualização. Por exemplo, os nossos resultados com luminância baixa comparados aos dados encontrados na literatura com luminância alta ((R. L. De Valois \& K. K. De Valois, 1988) mostram que a utilização de luminância baixa desloca a zona de máxima sensibilidade para a esquerda da FSC (i.e, para a freqüência de 0,5 cpg). No entanto, em níveis de luminância alta, a faixa de máxima sensibilidade ocorre por volta de 3,0 a 5,0 cpg (R. L. De Valois \& K. K. De Valois, 1988). Além disto, em níveis baixos de luminância, o SVH possivelmente não detecta freqüências espaciais acima de 2,4 cpg (Benedek et al., 2003). Por isto, que este trabalho se limitou a investigar freqüências espaciais entre 0,25 e 2,0 cpg. O deslocamento da sensibilidade máxima para freqüências mais baixas (p.ex., $0,5 \mathrm{cpg}$ ) pode estar relacionado ao funcionamento da via visual magnocelular que é especializada no processamento de freqüências espaciais baixas em níveis baixos de luminância (Benedek et al., 2003; Ellemberg et al., 1999; Schefrin et al., 1999; Kackson, Owsley, Cordle \& Finley, 1998). Entretanto, a FSC obtida neste trabalho é o resultado da interação entre o sistema parvocelular e magnocelular.

Novos estudos precisam investigar melhor a relação entre FSC e envelhecimento em condições mesópicas e escotópicas, utilizando método psicofísico semelhantes. Pois, trabalhos relacionando envelhecimento e FSC em condições escotópicas são raros (Schefrin et al., 1999) e não encontramos nenhum estudo comparando envelhecimento e FSC em condições fotópicas, mesópicas e escotópicas. Além disto, os estudos têm utilizado métodos psicofísicos diferentes e os mesmos poderão não gerar resultados totalmente semelhantes, pois existem, dentre outros aspectos, dificuldades com a variabilidade na estimação da sensibilidade ao contraste (Billock \& Harding, 1996). Além do mais, entende-se que qualquer método simples pode ter limitações, até porque o mesmo depende de interações espaço-temporais que são diferentes em cada caso (Benedek et al., 2003; Ellemberg et al., 1999). Entretanto, os nossos achados sugerem que o envelhecimento humano altera a percepção visual de contraste (FSC) até mesmo em níveis baixos de luminância.

\section{Referências}

Adams, R. J., \& Courage, M. L. (2002). Using a single test to measure human contrast sensitivity from early childhood to maturity. Vision Research, 42, 1205-1210.

Akutsu, H., \& Legge, G. E. (1995). Discrimination of compound gratings: Spatial-frequency channels or local features? Vision Research, 35, 2685-2695.

Arden, G. B. (1978). The importance of measuring contrast sensitivity in cases of visual disturbance. British Journal of Ophthalmology, 62, 198-209.

Arden, G. B., \& Jacobsen, J. (1978). A simple grating test for contrast sensitivity: Preliminary results indicate value for screening in glaucoma. Investigative Ophthalmology E Visual Science, 17, 23-32. 
Arundale, K. (1978). An investigation into the variation of human contrast sensitivity with age and ocular pathology. British Journal of Ophthalmology, 62, 213-215.

Benedek, G., Benedek, K., Kéri, S., \& Janáky, M. (2003). The scotopic low-frequency spatial contrast sensitivity develops in children between the ages of 5 and 14 years. Neuroscience Letters, 345, 161-164.

Billock, V., \& Harding, T. (1996). Evidence of spatial and temporal channels in the correlational structure of human spatialtemporal contrast sensitivity. Journal of Physiology, 490(2), 509-517.

Bour, L. J., \& Apkarian, P. (1996). Selective broad-band spatial frequency loss in contrast sensitivity functions. Investigative Ophthalmology \& Visual Science, 37(12), 2475-2484.

Braddick, F. W., Campbell, F. W., \& Atkinson, J. (1978). Channels in vision: Basic aspects. In R. Held, H. W. Leibowitz \& H. L. Teuber (Eds.), Handbook of sensory Physiology: Vol. 5. Perception (pp. 3-38). New York: Springer-Verlag.

Campbell, F. W., \& Robson, F. G. (1968). Application of the Fourier analysis to the visibility of gratings. Journal of Physiology, 197, 551-566.

Candy, T. R., Crowell, J. A., \& Banks, M. S. (1998). Optical, receptoral, and retinal constraints on foveal and peripheral vision in the human neonate. Vision Research, 38, 3857-3870.

Charman, W. N. (1996). Night Myopia and driving. Ophthalmic and Physiological Optics, 16(6), 474-478.

Courage, M. L., \& Adams, R. J. (1996). Infant peripheral vision: The development of monocular visual acuity in the first 3 months of postnatal life. Vision Research, 36, 1207-1215.

Crassini, B., Brown, B., \& Bowman, D. (1988). Age-related changes in contrast sensitivity in central and peripheral retina. Perception, 17, 315-332.

Derefeldt, F. D., Lennerstrand, G., \& Lundh, B. (1979). Age variations in normal human contrast sensitivity. Acta Ophthalmology, 57, 679-690.

De Valois, R. L., \& De Valois, K. K. (1988). Spatial vision. New York: Oxford University Press.

Ellemberg, D., Lewis, T. L., Liu, C. H., \& Maurer, D. (1999). Development of spatial and temporal vision during childhood. Vision Research, 39, 2325-2333.

Elliott, D. B. (1987). Contrast sensitivity decline with ageing: A neural or optical phenomenon? Ophthalmic and Physiological Optics, 7, 415-419.

Elliott, D. B., \& Situ, P. (1998). Visual acuity versus letter contrast sensitivity in early cataract. Vision Research, 38, 2047-2052.

Elliott, D, Whitaker, D., \& MacVeigh, D. (1990). Neural contribution to spatiotemporal contrast sensitivity decline in healthy ageing eyes. Vision Research, 30, 541-547.

Fiorentini, A., Porciatti, V., Morrone, M. C., \& Burr, D. C. (1996). Visual ageing: Unspecific decline of the responses to luminance and colour. Vision Research, 3, 3557-3566.

Georgeson, M. A., \& Sullivan, G. D. (1975). Contrast Constancy: Deblurring in human vision by spatial frequency channels. Journal of Physiology, 252, 627-656.

Graham, N. (1972). Spatial frequency channels in the human visual system: Effects of luminance and pattern drift rate. Vision Research, 12, 53-68.

Graham, N., \& Nachmias, J. (1971). Detection of grating patterns containing two spatial frequencies: A comparison of singlecannel and multiple channel models. Vision Research, 11, 251259.
Higgins, K. E, Jaffe, M. J., Caruso, R. C., \& Monasterio, F. M. de (1988). Spatial contrast sensitivity: Effects of age, test-retest, and psychophysical method. Journal of the Optical Society of America A, 5, 2173-2180.

John, R. S. (1997). Contrast detection and orientation discrimination thresholds associated with meridional amblyopia. Vision Research, 37, 1451-1457.

Kackson, G. R., Owsley, C. Cordle, E. P., \& Finley, D. F. (1998). Aging and scotopic sensitivity. Vision Research, 38, 3655-3662.

Kiper, D. C., \& Kiorpes, A. (1994). Suprathreshold contrast sensitivity in experimentally strabismic monkeys. Vision Research, 34, 1575-1583.

McGrath, C., \& Morrison, J. D. (1981). The effects of age on spatial frequency perception in human subjects. Quarterly Journal of Experimental Physiology, 66, 253-261.

Montés-Micó, R., Espana, E., Bueno, I., Charman, W. N., \& Menezo, J. L. (2004). Visual performance with multifocal intraocular lenses: Mesopic contrast sensitivity under distance and near conditions. Ophthalmology, 111 (1), 85-96.

Montés-Micó, R., \& Ferrer-Blasco, T. (2001). Contrast sensitivity function in children: Normalized notation for the assessment and diagnosis of diseases. Documenta Ophthalmologica, 103, 175-186.

Nio, Y. K., Jansonius, N. M., Fidler, V., Geraghty, E., Norrby, S., \& Kooijman, A. C. (2000). Age-related changes of defocusspecific contrast sensitivity in healthy subjects. Ophthalmic and Physiological Optics, 20, 323-334.

O`Donnell, B. F., Nestor, P. G., Potts, G. F., Stylianopoulos, K. C., Shenton, M. E., \& McCarley, R. W. (2002). Spatial frequency discrimination in schizophrenia. Journal of Abnormal Psychology, $111(4)$, 620-625.

Owsley, C., Sekuler, R., \& Siemsen, D. (1983). Contrast sensitivity throughout adulthood. Vision Research, 23, 689-699.

Richman, J. E., \& Lyons, S. (1994). A forced choice procedure for evaluation of contrast sensitivity function in preschool children. Journal of the American Optometric Association, 65(12), 859-864.

Ross, J. E., Clarke, D. D., \& Bron, A. J. (1985). Effect of age on contrast sensitivity function: Uniocular and binocular findings. British Journal of Ophthalmology, 69, 51-56.

Santos, N. A., Nogueira, R. M. T. L., \& Simas, M. L. B. (2005). Processamento visual da forma: Evidências para canais múltiplos de freqüências angulares em humanos. Psicologia Reflexão e Crítica, 18(1), 98-103.

Santos, N. A., \& Simas, M. L. B. (2002). Percepção e processamento visual da forma em humanos: Filtros de frequiências radiais de 1 e 4 cpg. Psicologia: Reflexão e Crítica, 15(2), 383-391.

Santos, N. A., Simas, M. L. B., \& Nogueira, R. M. T. B. L. (2003). Processamento visual da forma em idosos: Curvas de limiar de contraste para freqüências angulares e senoidais. Psicologia: Reflexão e Crítica, 16 (2), 271-277.

Santos, N. A., Simas, M. L. B., \& Nogueira, R. M. T. B. L. (2004). Comparison of angular frequency contrast sensitivity in young and older adults. Brazilian Journal of Medical and Biological Research, 37, 375-378.

Schefrin, B. E., Tregear, S. J., Harvey Jr, L. O., \& Werner, J. S. (1999). Senescent changes in scotopic contrast sensitivity. Vision Research, 39, 3728-3736.

Sekuler, R., Hutman, L. P., \& Owsley, C. (1980). Human aging and spatial vision. Science, 209, 1255-1256.

Simas, M. L. S., \& Santos, N. A. (2002a). Narrow-band 1, 2, 3, 4, 8, 16 and 24 cycles $/ 360^{\circ}$ angular frequency filters. Brazilian Journal of Medical and Biological Research, 35, 243-253. 
Simas, M. L. S., \& Santos, N. A. (2002b). Contrast sensitivity to radial frequencies modulated by Jn and jn Bessel profiles. Brazilian Journal of Medical and Biological Research, 35, 1357-1366.

Skalka, H. W. (1980). Effect of age on Arden grating acuity. British Journal of Ophthalmology, 64, 21-23.

Slaghuis, W. L., \& Thompson, A. K. (2003). The effect of peripheral visual motion on focal contrast sensitivity in positive- and negative-symptom schizophrenia. Neuropsychologia, 41, 968-980.

Sokol, S., Domar, A., \& Moskowitz, A. (1980). Utility of Arden grating test in glaucoma screening: High falsa-positive rate in normals over 50 years of age. Investigative Ophthalmology and Visual Science, 19, 1529-1533.

Suttle, C. M., \& Tumer, A. M. (2004). Transient pattern visual evoked potentials in children with Down's syndrome. Ophthalmic and Physiological Optics, 24, 91-99.

Van de Grind, W. A., Koenderink, J. J., \& Van Doorn, A. J. (2000). Motion detection from photopic to low scotopic muninance levels. Vision Research, 4O, 187-199.

Van Sluyters, R. C., Atkinson, M. S., Held, R. M., Hoffman, K., \& Shatz, C. J. (1990). The development of vision and visual perception. In S. W. Spillmann \& J. S. Werner (Eds.), The Neurophysiological Foundations (pp. 349-379). New York: Academic Press.

Ventura, D. F., Simões, A. L., Tomaz, S., Costa, M. F., Lago, M., Costa, M. T. V., Canto-Pereira, L. H. M., de Souza, J. M., Faria, M. A. M., \& Silveira, L. C. L. (2005). Colour vision and contrast sensitivity losses of mercury intoxicated industry workers in Brazil. Environmental Toxicology and Pharmacology, 19, 523-529.
Wetherill, G. B., \& Levitt, H. (1965). Sequential estimation of points on a psychometric function. The British Journal of Mathematical and Statistical Psychology, 48, 1-10.

Wilkinson, F., James, T. W., Wilson, H. R., Gati, J. S., Menon, E. S., \& Goodale, M. A. (2000). An fMRI study of the selective activation of human extrastriate form vision areas by radial and concentric gratings. Current Biology, 10, 1455-1458.

Wilson, H. R., \& Gelb, D. J. (1984). Modified line-element theory for spatial-frequency and width discrimination. Journal of the Optical Society of America A, 1(1), 124-131.

Wilson, H. R., Levi, D., Maffei, L., Rovamo, J., \& De Valois, R. (1990). The perception of form: Retina to striate cortex. In S W. Spillmann \& J. S. Werner (Eds.), Visual perception: The Neurophysiological Foundation (pp. 231-271). New York: Academic Press.

Wilson, H. R., McFarlane, D. K., \& Phillips, G. C. (1983). Spatial frequency tuning of orientation selective units estimated by oblique masking. Vision Research, 23(9), 873-882.

Woods, R. L., Tregear, S. J., \& Mitchell, R. A. (1998). Screening for ophthalmic disease in older subjects using visual acuity and contrast sensitivity. Ophthalmology, 105(12), 2318-2326.

Recebido: 06/01/2005

$1^{a}$ revisãa: $11 / 05 / 2005$

Aceite final: 21/06/2005 